\title{
Slow modes of polarization in purple membranes
}

\author{
Dietmar Porschke \\ Max Planck Institut für biophysikalische Chemie, Am Fassberg 11, 37077 Göttingen, Germany. \\ E-mail: dpoersc@gwdg.de
}

Received 10th June 2003, Accepted 30th October 2003

First published as an Advance Article on the web 21st November 2003

The polarization of bacteriorhodopsin discs has been characterized by measurements of the electric dichroism induced by sinusoidal electric field pulses in the frequency range from 0.2 to $100 \mathrm{kHz}$ with field strengths up to $40 \mathrm{kV} \mathrm{m}^{-1}$. Analysis of the stationary dichroism by a disk model with a saturating induced dipole moment in the direction of the plane shows saturation of the induced dipole at low field strengths in the range around $10 \mathrm{kV} \mathrm{m}^{-1}$. The AC-polarizability $\alpha_{\mathrm{AC}}$ increases with decreasing frequency, whereas the saturation field strength decreases with decreasing frequency in most cases. DC-polarizabilities $\alpha_{\mathrm{DC}}$ were obtained from the stationary dichroism induced by DC pulses and analyzed by the orientation function for discs with a permanent dipole perpendicular to the plane and an induced dipole in the plane. $\alpha_{\mathrm{DC}}$ is always larger than $\alpha_{\mathrm{AC}}$-values; $\alpha$-values increase from $100 \mathrm{kHz}$ to DC by factors of 5 to 9 . These data demonstrate the existence of a spectrum of slow polarization processes extending over the $\mu \mathrm{s}$ - into the ms-time range. The increase of polarizability with decreasing frequency was observed in buffers containing $\mathrm{Na}^{+}, \mathrm{Ca}^{2+}$ and $\mathrm{Mg}^{2+}$ as counterions. The dichroism rise curves observed under AC-pulses could be described by single exponentials $\tau^{r}$ in the absence of $\mathrm{Ca}^{2+}$ or $\mathrm{Mg}^{2+}$; $1 / \tau^{r}$ increases linearly with the square of the electric field strength, as expected for induced dipoles. The polarizabilities obtained from the slope of this dependence increase with decreasing frequency; the absolute values are higher by factors of $\sim 2$ than those derived from stationary dichroism data. The dichroism risecurves

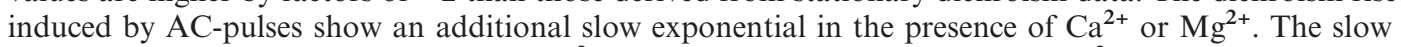
amplitude observed in the presence of $\mathrm{Mg}^{2+}$ is higher than in the presence of $\mathrm{Ca}^{2+}$, suggesting a contribution from $\mathrm{Mg}^{2+}$-inner sphere complexes. Thus, polarization data provide information about the coordination state of counterions. A simple criterion for the limit mechanisms of polarization by "biased dissociation" and "motion along surface" is discussed.

\section{Introduction}

Electric parameters of membranes and of membrane proteins are essential for understanding of bioelectric processes. ${ }^{1-3} \mathrm{~A}$ favored model system for analysis has been bacteriorhodop$\sin ,{ }^{4-7}$ because it is easily available and relatively stable. Its high anisotropy both with respect to electric and optical parameters $^{8-18}$ simplifies experimental characterization by electro-optical methods. ${ }^{19,20}$ A standard approach to determination of electric parameters requires measurements of electrooptical data (dichroism, birefringence or light scattering) using both high frequency and DC-pulses. ${ }^{9-11,14,15,17}$ The data at high frequencies are used to evaluate the polarizability, which is then introduced into the analysis of data obtained by DC pulses for determination of permanent dipole moments. This procedure is based on the assumption that the polarizability is independent of the frequency.

Polarization of ion atmospheres around polyelectrolytes involves rearrangement of the ion distribution ${ }^{21-34}$ and, thus, must be associated with some time distribution of ion motion at given field strengths. The time distribution of polarization may be dependent on the size of the object and on the field strength. Furthermore, the type of ion binding may affect the polarization time: for example, inner sphere complexes with long lifetimes ${ }^{35}$ are candidates for slow contributions to polarization.

Slow polarization processes have been mentioned in the literature, ${ }^{36,37}$ but the experimental evidence is quite limited in most cases. Dispersion curves of electro-optical amplitudes for bacteriorhodopsin suggest that the main relaxation of polarization occurs at about $1 \mathrm{MHz}$. Most dispersion curves shown in the literature ${ }^{10,15,37,38}$ indicate a plateau region between $\sim 100 \mathrm{kHz}$ and $\sim 100 \mathrm{~Hz}$ followed by the range of rotational relaxation at frequencies $<100 \mathrm{~Hz}$. Thus, polarizabilities measured at any frequency in the plateau region were assumed to be representative for bacteriorhodopsin.

In the present investigation, the dynamics of polarization in bacteriorhodopsin discs has been investigated in more detail by measurements of field strength dependences in the frequency domain from $100 \mathrm{kHz}$ to DC. The data clearly demonstrate a continuous increase of the polarizability with decreasing frequency $\nu$ in the range from $\nu<100 \mathrm{kHz}$ to DC. These results reveal a broad spectrum of polarization time constants.

\section{Structure and electric parameters of purple membranes}

The local and global structure of purple membranes is well known. $^{4-7}$ The membrane fragments prepared by standard procedures are obtained as thin disks with average diameters in the range of $1 \mu \mathrm{m}$. Membrane fragments with $1 \mu \mathrm{m}$ diameter include $\sim 6.8 \times 10^{4}$ protein monomers. Each of the protein monomers is surrounded by 10 lipids molecules with phosphate headgroups bearing negative charges. Thus, these fragments have a very high charge density and attract large numbers of counterions.

Electro-optical studies including measurements of the electric dichroism, birefringence and light scattering ${ }^{8-18}$ demonstrated that purple membranes have a large permanent dipole moment in the direction perpendicular to the disk plane and a large induced dipole moment along the disk plane (Fig. 1). The permanent dipole is dominant in the range of low DC fields. Because the energy of the induced dipole increases with the square of the field strength, the induced dipole takes over at high field strengths. The rotational diffusion 


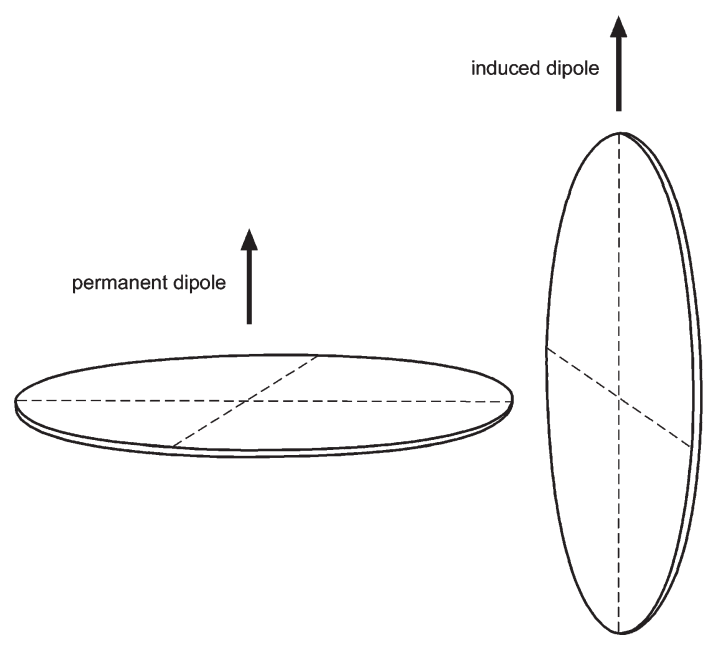

Fig. 1 Directions of dipole moments and modes of orientation of purple membranes.

of standard purple membrane fragments is relatively slow and, thus, the permanent dipole cannot follow rapid changes in the field direction. In contrast, field-induced redistribution of ions along the disk is relatively fast, resulting in orientation by the induced dipole at AC frequencies above $\sim 200$ $\mathrm{Hz}$. The orientation mechanism is indicated by the sign of the dichroism: at low DC fields the dichroism is negative, whereas at high DC fields and AC fields of sufficiently high frequency the dichroism is positive.

The nature of the permanent dipole moment has been characterized by a comparison ${ }^{39}$ of the $\mathrm{pH}$-dependence of experimental values with calculations based on the crystal structure. These data indicate that a high intrinsic dipole of the protein $(\sim 600 \mathrm{D}$ per monomer, calculated for the transmembrane direction) is compensated to a large degree at neutral $\mathrm{pH}$ (experimental value $\sim 53 \mathrm{D}$ per monomer). The compensation seems to be due to a non-symmetric distribution of charges on lipid headgroups. According to the available crystal structure there are 5 lipid headgroups per protein monomer on each side of the membrane. ${ }^{40}$ Thus, the nonsymmetric distribution of charges suggests a difference in the charge state of some headgroup residue(s).

The induced dipole is attributed to field induced polarization of the ion atmosphere in the disk plane. Details of the polarization mechanism are analyzed in the discussion.

\section{Orientation function}

The field induced orientation of disk structures with an induced dipole in the disk plane and a permanent dipole perpendicular to the disk plane has been described by Shah. ${ }^{42}$ He assumed a standard induced dipole in the disk plane and did not consider saturation of the polarizability. Because the experimental data indicate saturation in the case of bacteriorhodopsin discs ( $c f$. below), the orientation function has to be extended.

Saturation of polarizability has been described by various functions. It is expected that the standard induced dipole found at low field strengths with an energy

$$
U_{i}=-\frac{1}{2} \alpha E^{2}(\sin \theta)^{2}
$$

( $\alpha$ polarizability, $E$ field strength, $\theta$ orientation angle) is followed by a transition regime before the polarization is saturated. A function satisfying this requirement ${ }^{43}$ is given by

$$
U_{i}=-\alpha E_{0}^{2}\left[\sqrt{1+\left(E \sin (\theta) / E_{0}\right)^{2}}-1\right]
$$

where $E_{0}$ is the saturation field strength. At high field strengths $E \gg E_{0}$ the energy is given by

$$
U_{i}=-\alpha E_{0} E \sin (\theta)
$$

whereas at low field strengths $E \ll E_{0}$ the energy corresponds to that of eqn. (1). The full orientation function, including the contribution resulting from the permanent dipole moment $\mu$ (cf. Shah), is given by

$$
\Phi=\frac{\int_{0}^{\pi} B \frac{3 \cos ^{2}(\phi)-1}{2} 2 \pi \sin (\phi) \mathrm{d} \phi}{\int_{0}^{\pi} B 2 \pi \sin (\phi) \mathrm{d} \phi}
$$

where $B=\exp \left[\left(\mu E \cos \phi+\alpha E_{0}^{2}\left[\left(1+\left[E \cos (\phi) / E_{0}\right]^{2}\right)^{1 / 2}-1\right]\right) / k T\right]$. This orientation function approaches a value $-\frac{1}{2}$ at high field strengths. Thus, the dichroism at any field strength is described by

$$
\xi=-2 \Phi \xi_{\infty}
$$

where $\xi_{\infty}$ denotes the limiting value of the dichroism at high field strengths. For fitting of parameters to experimental data, the orientation function (5) was solved by numerical procedures.

\section{Materials and methods}

Purple membranes were isolated from Halobacterium salinarum $S 9$ (given by Dr J. Tittor) using standard procedures, including fractionation by sucrose density centrifugation. ${ }^{41}$ The samples proved to be homogeneous by SDS-PAGE and by isoelectric focusing. The D96N-sample was given by Dr J. Tittor. Before measurements, the membrane suspensions were extensively dialyzed against standard $\mathrm{pH}$ buffers and were sonicated in a Bransonic 220 for 3 min to break down aggregates. The following standard buffers were used: $1 \mathrm{mM}$ $\mathrm{NaClO}_{4}, 1 \mathrm{mM}$ Na-cacodylate $\mathrm{pH} 7,0.2 \mathrm{mM}$ EDTA (NCE); $1 \mathrm{mM} \mathrm{NaClO} 4,1 \mathrm{mM}$ sodium cacodylate $\mathrm{pH}$ 7,

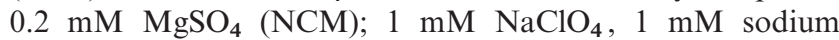
cacodylate $\mathrm{pH}$ 7, $0.2 \mathrm{mM} \mathrm{CaClO}_{4}$ (NCC).

The electric dichroism was measured at $546 \mathrm{~nm}$ and $20^{\circ} \mathrm{C}$ using a setup described previously ${ }^{18}$ (Fig. 2). Electric field pulses were generated by a Tektronix AWG 5105 arbitrary waveform generator together with a Fluke 5205A amplifier. A Fluke 5700A Calibrator combined with a relay device for adjusting pulse lengths was also used for pulse generation. The electric field pulses were applied to the samples in a cuvette with $1 \mathrm{~cm}$ optical pathlength and with an insert machined from Teflon holding platinum electrodes at a distance of $4.8 \mathrm{~mm}$. The absorbance at $546 \mathrm{~nm}$ was in the range of 0.1 to 0.15 . Usually the plane of polarization was adjusted parallel to the field vector. In order to maintain the light-adapted state of $\mathrm{BR}$, samples were exposed to the light of a $100 \mathrm{~W}$ lamp. Complete light-adaptation of BR was monitored by determining the ratio of absorbances at $590 \mathrm{~nm}$ and $540 \mathrm{~nm}$. This ratio is about 1.04 for light-adapted bacteriorhodopsin. Sufficiently long electric field pulses were applied to ensure complete adjustment of the stationary state of orientation. Stationary

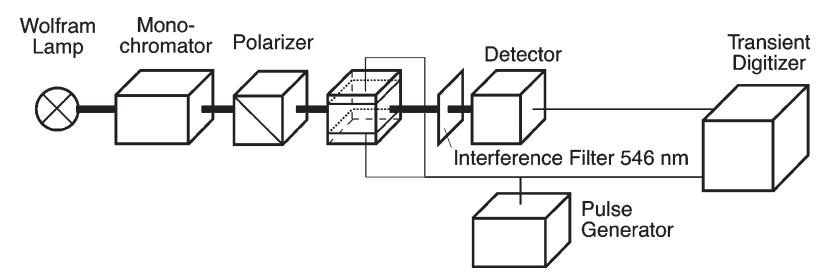

Fig. 2 Scheme of the instrument used for measurements of the electric dichroism. 
values of the dichroism were calculated from the stationary change of light intensity using the standard equation:

$$
\xi=\frac{\Delta A_{\|}-\Delta A_{\perp}}{\bar{A}}=\frac{1.5 \Delta A_{\|}}{\bar{A}}
$$

where $\Delta A_{\|}$and $\Delta A_{\perp}$ are the field-induced absorbance changes determined by light polarized parallel and perpendicular to the field vector, respectively. $\bar{A}$ is the absorbance in the absence of an external electric field. For fitting of experimental data a simplex routine was used. The parameters in Table 1 were obtained by least squares fits of the dichroism ( $c f$. Fig. 4) with a minimum in the sum of absolute residuals.

\section{Results}

\section{Stationary AC dichroism}

AC electric field pulses applied to solutions of bacteriorhodopsin induce positive dichroism. Dispersion curves of this effect shown in the literature are restricted to single values of the electric field strength. A set of measurements at different field strengths is given in Fig. 3a in the standard form of the reduced electric dichroism. When these data are normalized to 1 at the maximal values of the dichroism observed at each field strength separately ( $c f$. Fig. 3b), the resulting dispersion curves reveal a clear dependence of the shape on the field strength that has not been described in the literature yet. The distinct dependence on the frequency at different field strengths is also clearly illustrated in a set of data obtained for a solution containing $\mathrm{Ca}^{2+}$-ions (Fig. 3c).

Stationary values of the dichroism $\xi$ measured as a function of the field strength $E$ at a given frequency can be described by the standard orientation function for induced dipoles, ${ }^{19}$ if the data are restricted to low field strengths $E \leq 5 \mathrm{kV} \mathrm{m}^{-1}$. Experimental data measured over a wider range of field strengths require an extended orientation function for a satisfactory description. As shown in Fig. 4, the orientation function with saturation of the polarizability presented above can be used to describe the experimental data at a high accuracy. Because the permanent dipole of bacteriorhodopsin does not contribute under the applied AC pulses, its value was fixed to $\mu=0$ during fitting with eqn. (5).

The dichroism data measured at the highest field strengths $E \sim 40 \mathrm{kV} \mathrm{m}^{-1}$ are close to the limiting value ( $c f$. Fig. $4 \mathrm{a}$ ), and, thus, fitted values of the limit dichroism can be considered as reliable. Furthermore, the dichroism values $\xi$ observed at different frequencies approach very similar limiting values at high field strengths. However, the dichroism $\xi$ at low field strengths clearly increases with decreasing frequency ( $c f$. Fig. $4 \mathrm{~b}$ ), reflecting an increase of the polarizability with decreasing frequency. Least-squares fits of the data by the orientation function provided values for the polarizability, the saturation field strength and the limiting value of the dichroism compiled in Table 1. The polarizabilities measured both for wild type bacteriorhodopsin and for the mutant D96N at different ionic conditions increase with decreasing frequency. The saturation field strength $E_{0}$ decreases with decreasing frequency for the D96N-sample. Most of the $E_{0}$-values obtained for the wild type sample show a similar trend-with the exception of the data at $0.2 \mathrm{kHz}$.

\section{AC-dichroism risecurves}

An independent method for determination of polarizabilities is based on the field strength dependence of the risetime constants. In the absence of $\mathrm{Mg}^{2+}$ or $\mathrm{Ca}^{2+}$, secured by dialysis against EDTA, the dichroism risecurves can be described by single exponentials at a high accuracy. According to the theory for induced dipoles, ${ }^{44}$ the rise time constants $\tau^{r}$ should be dependent on the electric field strength $E$ according to

$$
\frac{1}{\tau^{r}}=\frac{2 \alpha D_{\mathrm{r}}}{x k T} E^{2}=\frac{\alpha}{3 x k T \tau_{0}^{r}} E^{2}
$$

where $k T$ is the thermal energy, $D_{\mathbf{r}}=1 /\left(6 \tau_{0}^{r}\right)$ the rotational diffusion coefficient, $\tau_{0}^{r}$ the dichroism decay time and $x$ a numerical factor that was specified by Schwarz $^{44}$ as 3.35 , whereas simulations of Brownian dynamics ${ }^{45}$ for rod-like molecules demonstrated a value $x=2.9$. Plots of $1 / \tau^{r}$ against $E^{2}$ show that eqn. (7) is valid also in the present case $(c f$. Fig. $5)$; the slope $\mathrm{d}\left(1 / \tau^{r}\right) / \mathrm{d}\left(E^{2}\right)$ increases with decreasing frequency, demonstrating an increase of the polarizability with decreasing frequency. The $\alpha$-values obtained with $x=2.9$ and $\tau_{0}^{r}=91.8 \mathrm{~ms}$ are $3.48,4.68,6.18$ and 6.60 in units of $\left[10^{-28} \mathrm{C} \mathrm{m}^{2} \mathrm{~V}^{-1}\right]$ for $100,10,1$ and $0.2 \mathrm{kHz}$, respectively. Thus, the polarizabilities obtained from the risetimes constants reveal the same dependence on the frequency as that derived from the stationary values of the dichroism, but the latter values are lower by a factor of about 2 .

The present investigation of the frequency dependence was not extended below $\nu=0.2 \mathrm{kHz}$ because of $(2 \nu)$-modulation of the dichroism curves at $\nu<0.2 \mathrm{kHz}$. The effect is already clearly visible at $0.2 \mathrm{kHz}$ at higher field strengths, where orientational relaxation into the direction of the field vector is accelerated. Deviations of relaxation time constants observed at 0.2 $\mathrm{kHz}$ at high field strengths ( $c f$. two points not included in the fit in Fig. 5) seem to be due to this effect.

In the presence of $\mathrm{Ca}^{2+}-$ or $\mathrm{Mg}^{2+}$-ions the dichroism rise curves cannot be described by single exponentials, but require a second exponential for a reasonable description ( $c f$. Fig. 6). The reciprocal time constant of this second exponential can be represented as a linear function of the square of the electric field strength at a reasonable accuracy. The slope of this dependence is about a factor of 10 smaller than that found for the single exponential described above, suggesting a smaller contribution to the polarizability. In general the amplitude is larger in the presence of $\mathrm{Mg}^{2+}$ than in the presence of $\mathrm{Ca}^{2+}$, which may come from a contribution from $\mathrm{Mg}^{2+}$-inner sphere complexes.

The dichroism curves were measured at different orientations of the polarizer, in particular at the magic angle orientation. These measurements did not provide evidence for the

Table 1 Electro-optical parameters of wild-type (WT) and D96N bacteriorhodopsin measured at different frequencies $\nu$ in various buffers

\begin{tabular}{|c|c|c|c|c|c|c|c|c|c|c|c|c|c|c|c|c|}
\hline & \multirow[b]{2}{*}{$\nu$} & \multicolumn{5}{|c|}{$10^{28} \alpha / \mathrm{C} \mathrm{m}^{2} \mathrm{~V}^{-1}$} & \multicolumn{4}{|c|}{$E_{0} / \mathrm{kV} \mathrm{m}^{-1}$} & \multirow{2}{*}{$\begin{array}{l}10^{24} \mu / \mathrm{C} \mathrm{m} \\
\mathrm{DC}\end{array}$} & \multicolumn{5}{|l|}{$\xi$} \\
\hline & & 100 & 10 & 1 & 0.2 & $\mathrm{DC}$ & 100 & 10 & 1 & 0.2 & & 100 & 10 & 1 & 0.2 & $\mathrm{DC}$ \\
\hline \multirow[t]{3}{*}{ WT } & $\mathrm{NCE}$ & 1.60 & 2.12 & 2.82 & 3.32 & 7.92 & 11.8 & 10.6 & 9 & 11 & 7.7 & 0.505 & 0.504 & 0.493 & 0.443 & 0.268 \\
\hline & $\mathrm{NCC}$ & 1.57 & 2.14 & 3.12 & 3.57 & 7.52 & 10.3 & 9 & 8.9 & 11.9 & 7.45 & 0.290 & 0.291 & 0.268 & 0.237 & 0.118 \\
\hline & NCM & 1.12 & 1.57 & 2.48 & 2.96 & 8.90 & 9.8 & 8.8 & 7.8 & 10.1 & 7.89 & 0.273 & 0.272 & 0.247 & 0.207 & 0.064 \\
\hline \multirow[t]{3}{*}{ D96N } & NCE & 0.64 & 0.86 & 1.24 & 2.49 & 3.29 & 13.3 & 10.9 & 10.3 & 6.3 & 11.8 & 0.385 & 0.376 & 0.300 & 0.174 & 0.274 \\
\hline & $\mathrm{NCC}$ & 0.67 & 0.98 & 1.59 & 2.44 & 3.90 & 13.8 & 13.1 & 12 & 11.2 & 10.5 & 0.185 & 0.172 & 0.139 & 0.096 & 0.094 \\
\hline & NCM & 0.58 & 0.85 & 1.52 & 2.38 & 5.33 & 14.7 & 12.5 & 10.9 & 9.2 & 11.5 & 0.184 & 0.179 & 0.138 & 0.097 & 0.089 \\
\hline
\end{tabular}
$\left(\alpha\right.$ polarizability, $E_{0}$ saturation field strength, $\mu$ permanent dipole moment, $\xi$ limiting value of the dichroism, $20^{\circ} \mathrm{C}$, estimated accuracy $\pm 10^{\circ} \%$ ) 

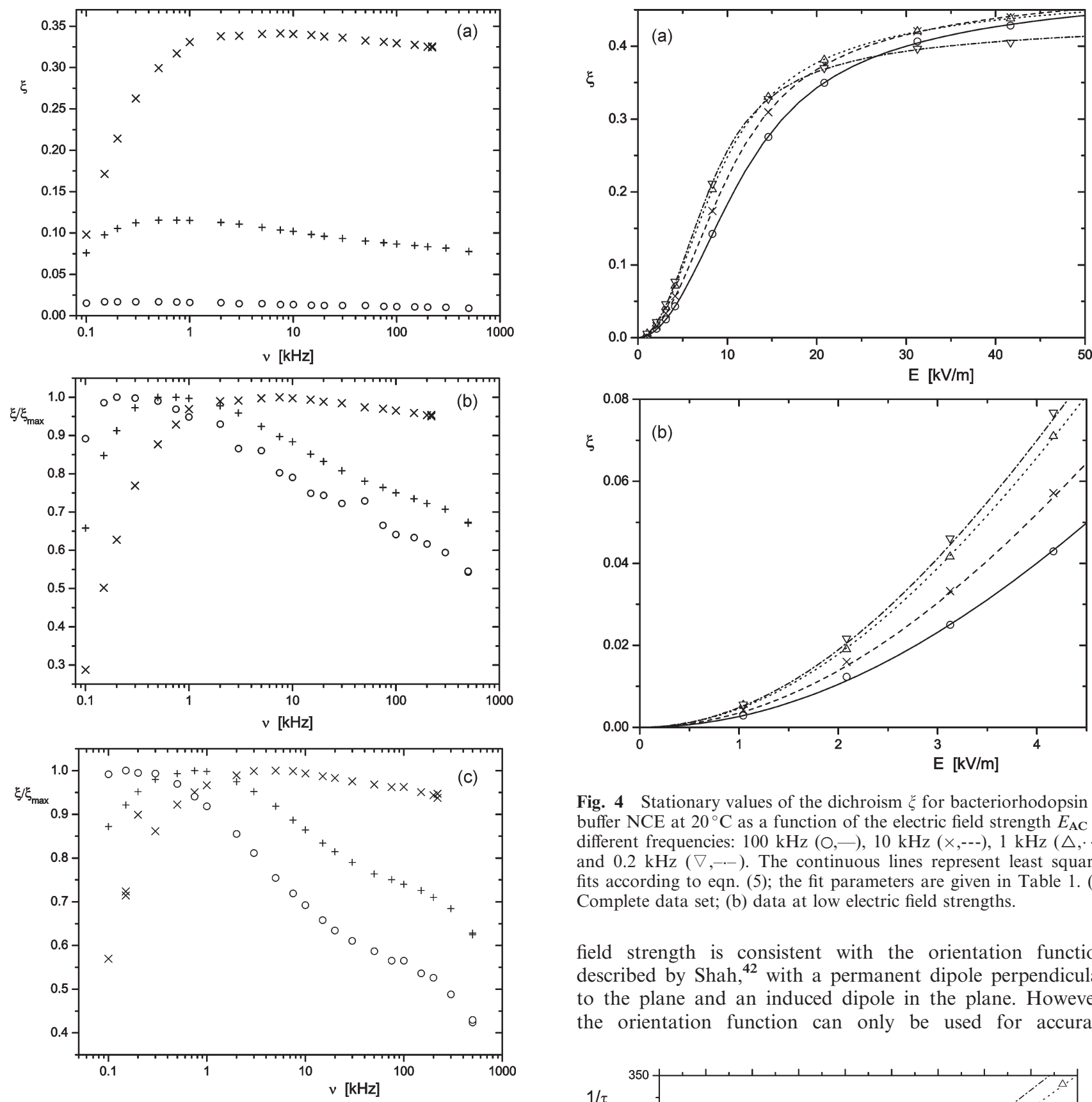

Fig. 3 (a) Stationary values of the dichroism $\xi$ for bacteriorhodopsin in buffer NCE at $20^{\circ} \mathrm{C}$ as a function of the frequency $\nu$ at different field strengths $E\left[\mathrm{kV} \mathrm{m}^{-1}\right]: 2.083(\mathrm{O}), 6.25(+)$ and $20.83(\times)$; (b) Same data as in (a) but normalized to unity at the maximal $\xi$-value measured at the given field strength, i.e. the $\xi$-values measured at each given field strength were divided by the maximal value $\xi_{\max }$ found at the given field strength; (c) data measured in buffer NCC, normalized as described in $b$. The symbols representing given field strengths in panels $\mathrm{b}$ and $\mathrm{c}$ are as defined in $\mathrm{a}$.

existence of field induced reactions occurring under the conditions of the present experiments. Thus, the slow relaxation effect observed in the presence of $\mathrm{Ca}^{2+}$ or $\mathrm{Mg}^{2+}$ is not due to field induced association of bacteriorhodopsin discs, which should be indicated by an increase of the absorbance due to turbidity.

\section{Stationary DC-dichroism}

The stationary dichroism measured under DC pulses is negative at low field strengths and turns to positive values at high field strengths. The general form of this dependence on the

Fig. 4 Stationary values of the dichroism $\xi$ for bacteriorhodopsin in buffer NCE at $20^{\circ} \mathrm{C}$ as a function of the electric field strength $E_{\mathrm{AC}}$ at different frequencies: $100 \mathrm{kHz}(\mathrm{O},-), 10 \mathrm{kHz}(\times,---), 1 \mathrm{kHz}(\triangle, \cdots)$ and $0.2 \mathrm{kHz}(\nabla,-\cdot-)$. The continuous lines represent least squares fits according to eqn. (5); the fit parameters are given in Table 1. (a) Complete data set; (b) data at low electric field strengths.

field strength is consistent with the orientation function described by Shah, ${ }^{42}$ with a permanent dipole perpendicular to the plane and an induced dipole in the plane. However, the orientation function can only be used for accurate

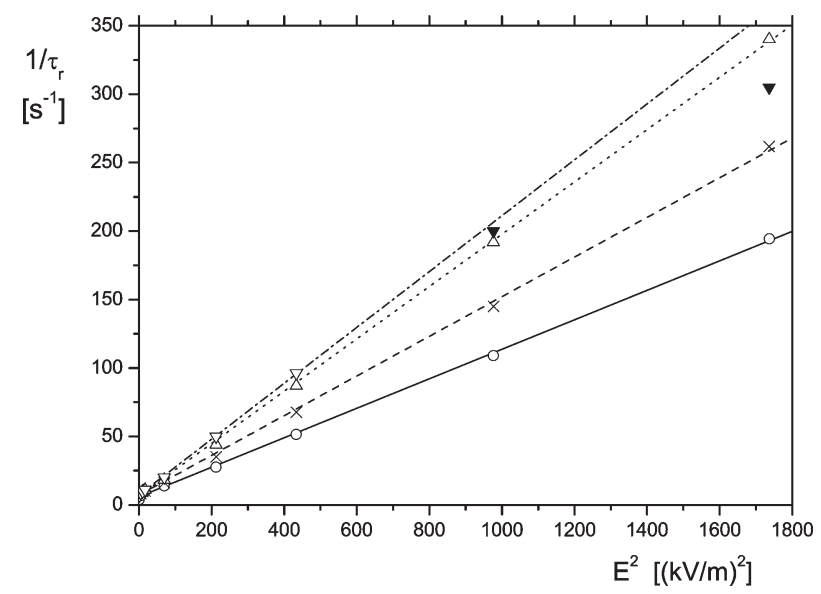

Fig. 5 Reciprocal values of dichroism rise time constants $1 / \tau^{r}$ for bacteriorhodopsin in buffer $\mathrm{NCE}$ at $20^{\circ} \mathrm{C}$ as a function of the square of the electric field strength $E^{2}$ at different frequencies: $100 \mathrm{kHz}(\mathrm{O},-)$, $10 \mathrm{kHz}(\times,---), 1 \mathrm{kHz}(\triangle, \cdots)$ and $0.2 \mathrm{kHz}(\nabla,-\cdot-)$. The data at 0.2 $\mathrm{kHz}$ denoted by " $\boldsymbol{\nabla}$ " indicate saturation of the polarizability and, thus, were not included in the linear regression. Using eqn. (7) with factor $x=2.9$ and $\tau_{0}^{r}=91.8 \mathrm{~ms}$, the slopes of the linear regressions provided the polarizabilities $3.48,4.68,6.18$ and 6.60 in units of $\left[10^{-28} \mathrm{C} \mathrm{m}^{2} \mathrm{~V}^{-1}\right]$ for $100,10,1$ and $0.2 \mathrm{kHz}$, respectively. 


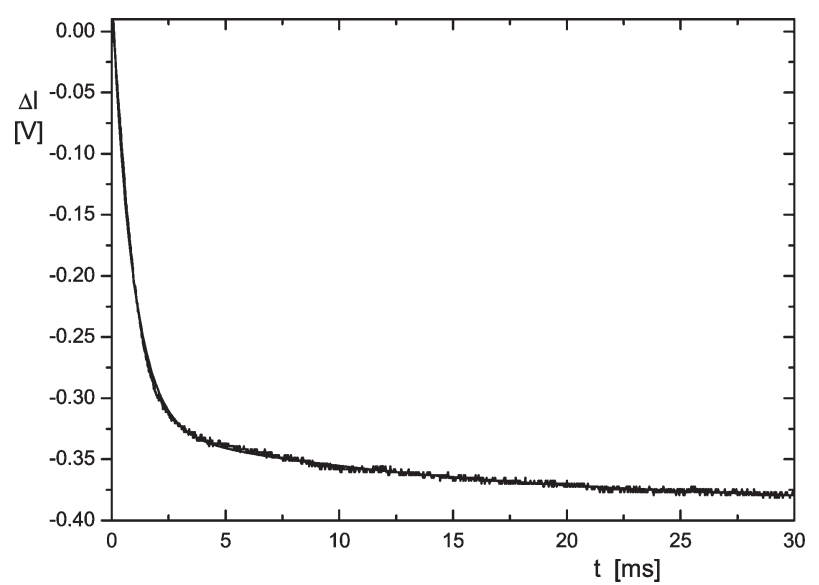

Fig. 6 Dichroism rise curve for bacteriorhodopsin in buffer NCM induced by a $10 \mathrm{kHz}$ pulse with an effective electric field strength of $73.7 \mathrm{kV} \mathrm{m}^{-1}$. A least squares fit provides the time constants $\tau_{1}=0.917 \mathrm{~ms}$ and $\tau_{2}=14.8 \mathrm{~ms}\left(20^{\circ} \mathrm{C}\right)$.

representation of data measured at low field strength. Deviations of experimental data at higher field strengths are considerable-it is not possible to fit data measured over a wide range of field strengths by the Shah function. ${ }^{18}$

The saturation of the polarizability observed under ACpulses is expected to occur also under DC-pulses and, thus, the orientation function, eqn. (5), should be useful for description of the stationary DC-dichroism as well. As shown in Fig. 7 the experimental data can be described by eqn. (5) at a high accuracy over the full range of electric field strengths. However, examination of the fitted parameters reveals a serious problem: the limit dichroism is extreme $(-2.98)$ and outside reasonable limits. Further analysis shows that the electric parameters are also not reasonable: the induced dipole term is dominant at low field strengths and the permanent dipole term prevails at high field strengths-just opposite to the conclusions derived from the shape of the experimental transients. ${ }^{18}$ Thus, it must be concluded that the parameters are not reasonable from the physical point of view, although the fit happens to be excellent.

The parameters obtained by least squares fitting of the data in Fig. 7 by eqns. (4) and (5) imply a special balance of the

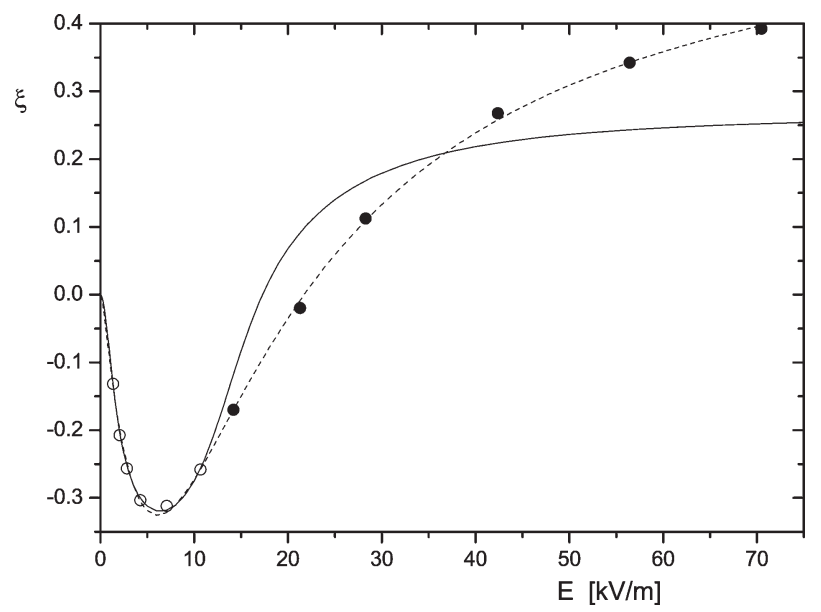

Fig. 7 Stationary values of the dichroism $\xi$ for bacteriorhodopsin in buffer NCE at $20^{\circ} \mathrm{C}$ as a function of the DC electric field strength $E_{\text {DC }}$. The data denoted by "O" were included in a least squares fit to the Shah orientation function, represented by the continuous line (fit parameters given in Table 1). The data denoted by "•", not included in the fit, demonstrate the deviations from the Shah function at high field strengths. The dashed line shows a least squares fit of the data by eqns. (4) and (5) with the parameters $\mu=7.83 \times 10^{-25} \mathrm{C} \mathrm{m}$, $\alpha=2.45 \times 10^{-27} \mathrm{C} \mathrm{m}^{2} \mathrm{~V}^{-1}, E_{0}=0.39 \mathrm{kV} \mathrm{m}^{-1}, \xi=-2.98$. energies from the induced and the permanent dipole up to high field strengths. Because these energies remain rather close to each other, the degree of orientation is far from saturation even at the highest field strength shown in Fig. 7. In contrast, the parameters obtained from the Shah orientation function lead to saturation of orientation at relatively low field strengths, because the energy of the induced dipole increases with the square of the field strength.

Under the given conditions, the results obtained by the Shah orientation function from the data at low field strengths must be considered as the best available approximation. The DC polarizabilities obtained by this procedure, compiled in Table 1, are higher than AC polarizabilities measured at $0.2 \mathrm{kHz}$. Thus, there is a continuous increase of the polarizability with decreasing frequency.

\section{Discussion}

\section{Modes of polarization}

A major part of the ions associated with purple membranes is expected to be in the form of a mobile ion atmosphere. This appears to be consistent with the experimental data in the literature suggesting that the main part of the polarization is a very fast process with a relaxation frequency in the $\mathrm{MHz}$ range. However, some of these ions are probably bound to the discs in a less mobile form and, thus, slower components of field induced redistribution may be expected. Potential sites for coordination of ions are phosphate groups of lipids and in addition carboxyl groups of aspartic or glutamic acids in the protein. The relaxation time constants of these ion complexes may cover a broad time range and, thus, a broad range of polarization time constants may be expected.

Although slow polarization processes in polyelectrolytes may be predicted to exist under certain conditions, experimental evidence is hardly available. This is partly due to the difficulty that most polymer samples are not suitable for a quantitative analysis because of flexibility and/or heterogeneity with respect to particle size. Both flexibility and heterogeneity lead to a broad spectrum of time constants for orientation and, thus, it is impossible to separate orientational from polarization processes. In the case of purple membranes, the flexibility is limited and the heterogeneity can be reduced to a tolerable level. For the sample used in the present investigation the heterogeneity is indicated by a spectrum of dichroism decay time constants in the range from $\sim 35$ to $\sim 150 \mathrm{~ms}$. The rotation time constant $\tau_{\mathbf{r}}$ of circular disks ${ }^{46}$ is given by

$$
\tau_{\mathrm{r}}=\frac{2 \eta d^{3}}{9 k T}
$$

where $d$ is the diameter, $\eta$ the viscosity and $k T$ the thermal energy. Thus, the range of time constants corresponds to a size distribution with diameters from $\sim 0.9$ to $\sim 1.4 \mu \mathrm{m}$, which is relatively narrow. This sample has been used to determine the AC polarizability over the frequency range from 100 to $0.2 \mathrm{kHz}$ (Table 1, Fig. 8). Moreover, the frequency range is extended by the determination of the DC-polarizability.

The experimental data indicate an unexpectedly broad distribution of polarization time constants. According to the present results, the influence of bivalent ions on the overall shape of the dispersion curves (Fig. 8) is relatively small. The spectrum of time constants extends from the $\mu$ s to the ms time range, both in the absence and presence of bivalent ions. Monovalent counterions like $\mathrm{Na}^{+}$are known for their high reaction rates and, thus, the physical basis for slow processes should be specified. In this context it is useful to compare the present data with results obtained for latex particles by dielectric relaxation. Schwan et al. ${ }^{47}$ demonstrated that the characteristic dielectric frequency of latex particles decreases 


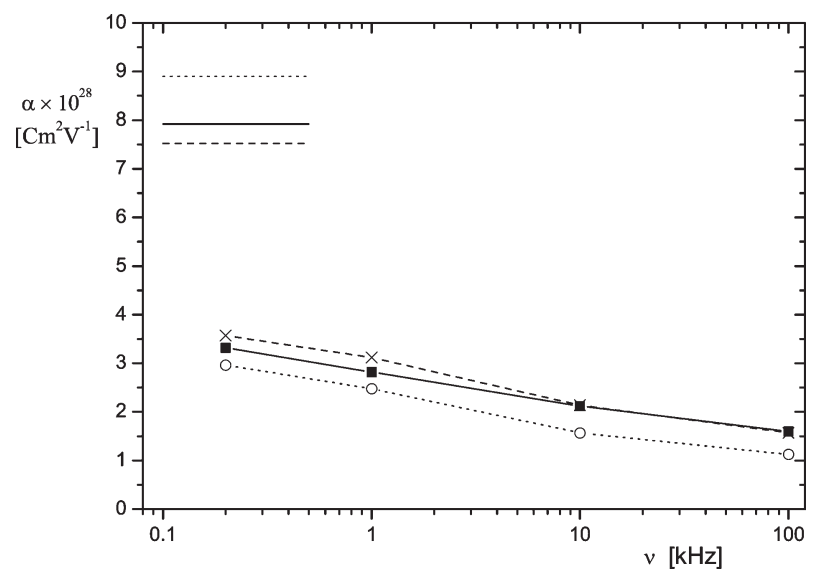

Fig. 8 Polarizability $\alpha$ of purple membranes as a function of the frequency $\nu$ in buffers $\operatorname{NCE}(\mathbf{\square},-), \operatorname{NCC}(\times,--)$ and $\operatorname{NCM}(0, \cdots)$. The polarizabilities obtained from DC-experiments are indicated by horizontal lines $\left(20^{\circ} \mathrm{C}\right)$.

with the square of their radius. This relation was found to be independent of the nature and the concentration of electrolyte as well as of the concentration of the particles. For particles with $1.17 \mathrm{~mm}$ diameter the characteristic frequency was $\sim 500 \mathrm{~Hz}$, with a "fairly well defined spectrum of relaxation times". Thus, the time constants of polarization are in the same range as those observed for purple membrane fragments. The different shape of latex particles and purple membranes may also have some influence on the spectrum of polarization times, but the influence of the size is expected to be more important than that of the shape.

The experimental data obtained by Schwan et al. ${ }^{47}$ were explained by a theory of Schwarz ${ }^{22}$ in terms of a displacement of counterions in the atmosphere around the latex particles. According to this theory the polarization time constant of spherical particles is given by

$$
\tau_{\mathrm{p}}=\frac{r^{2}}{2 D}
$$

where $r$ is the radius of the sphere and $D$ is the surface diffusion coefficient of the counterions. When the standard diffusion coefficient of counterions in aqueous solution is used, the resulting time constant is smaller than the experimental one. Thus, motion of counterions along the surface is slowed down by some activation barriers. By a spectrum of activation energies, Schwarz ${ }^{22}$ accounted for the relaxation spectrum indicated by the experimental dispersion curves.

The theory of Schwarz ${ }^{22}$ was criticized by Schurr ${ }^{23}$ and later by Fixman ${ }^{28}$ for omission of any flux of charge into, or out of, the bulk solution. Fixman concluded that the dominant slow process that controls relaxation is the slow diffusion of neutral salt in the environment of the macromolecule. The theoretical literature on the polarization of polyelectrolytes is extensive $^{21-32}$ and, thus, cannot be reviewed here. Apparently the polarization process is too complex for description by a tractable analytical theory. More information may be obtained by Brownian dynamics simulations. ${ }^{33,34}$

\section{Limit cases of polarization mechanisms}

In the absence of a comprehensive theory for polarization of polyelectrolytes it may be useful to discuss simple limit mechanisms. A dipole of any polyelectrolyte may be induced by motion of counterions along the polymer or by a nonsymmetric change of ion association at the ends of the polymer. Evidence for the latter mechanism was obtained in the case of short, rod-like DNA restriction fragments, ${ }^{48}$ where polarization was observed in the ns-time range. The reciprocal polarization time constants were shown to be dependent on the ion concentration as expected for a diffusion controlled association reaction. The experimental data are consistent with field-induced non-symmetric dissociation of ions at one end of the DNA. The driving force for this reaction is generation of the dipole. Evidence for non-symmetric dissociation has been obtained for restriction fragments with 76 and 95 base pairs. ${ }^{48}$ For these fragments the experimental data do not show any chain length dependence of the polarization time constants. The time constants of polarization found in the experiments were reproduced by simulations of Brownian dynamics. $^{34}$

The strong evidence for polarization by biased dissociation in the case of DNA double helices raises the question for a simple criterion to predict conditions, where the different limit mechanisms are expected to be active. The "biased dissociation" mechanism is characterized by the time constant of ion dissociation, whereas the "motion along surface" mechanism is characterized by the diffusion coefficient of ions along the surface $D_{\mathrm{i}}$. The time constant of dissociation is given by $1 /$ $k_{\mathrm{d}}$, where $k_{\mathrm{d}}$ is the rate constant of ion dissociation. During a time interval $1 / k_{\mathbf{d}}$, ions may move along the surface by a characteristic distance $d=\sqrt{ }\left(D_{\mathrm{i}} / k_{\mathrm{d}}\right)$. If the polymer is longer than $d$, polarization by "biased dissociation" is faster than by "motion along surface". Thus, the "biased dissociation" mechanism is expected to be dominant for objects with dimensions $>d$, whereas the "motion along surface" mechanism should be dominant for objects with dimension $<d$ ( $c f$. ref. 30 ). For DNA the rate constant $k_{\mathrm{d}}$ for monovalent ions ${ }^{48}$ at low salt concentration $(\sim 1 \mathrm{mM})$ is in the range $10^{7} \mathrm{~s}^{-1}$ and the diffusion coefficient for $\mathrm{Na}^{+}$in water is $1.3 \times 10^{-5} \mathrm{~cm}^{2} \mathrm{~s}^{-1}$. Using these values we get a value of $11 \mathrm{~nm}$ for the characteristic distance. Thus, the biased dissociation mechanism is expected to be effective for DNA's with more than $\sim 35$ base pairs, in agreement with the experiments. ${ }^{48}$ This criterion based on comparison of time constants should be useful as a simple approximation.

Application of the simple criterion to purple membranes indicates that there are rather slow processes of both ion dissociation and motion of ions along the surface. This conclusion is supported by independent experiments. Dissociation of $\mathrm{Mg}^{2+}$-ions induced by addition of EDTA was reflected by changes of electro-optical data with time constants in the range of hours up to days (in preparation). Although this slow process may be controlled by reactions within the protein, the slow reactions of the protein are then obviously coupled with ion binding.

Finally, application of the simple polarization criterion to the case of latex particles raises some questions. As shown by Schwarz, ${ }^{22}$ the observation of slow polarization processes is compatible with motion of ions along the surface, if some activation barriers of reasonable magnitude are assumed. If this interpretation is correct, dissociation of ions from the surface layer should be relatively slow, because otherwise the "biased dissociation" mechanism would be active.

\section{Binding state of bivalent ions}

The dispersion curves obtained in the presence of different ions are equivalent in a first approximation, but a more detailed analysis reveals some difference. The ratio of the polarizabilities $\alpha_{\mathrm{DC}} / \alpha_{100}$ measured at the extremes of the frequency range is 5.0 (5.1) in the absence of bivalent ions, 4.8 (5.8) in the presence of $\mathrm{Ca}^{2+}$ and 7.9 (9.2) in the presence of $\mathrm{Mg}^{2+}$ for wild-type bacteriorhodopsin (and for D96N, respectively). The high ratio observed in the presence of $\mathrm{Mg}^{2+}$ indicates a particularly high contribution from slow polarization processes, which seem to be mainly due to inner sphere complexes. It is well known that these complexes ${ }^{35}$ react slowly with a characteristic rate constant of $10^{5} \mathrm{~s}^{-1}$ for formation 
of inner sphere contacts and with relaxation time constants in the ms-time range. $\mathrm{Ca}^{2+}$ ions are very similar with respect to equilibrium parameters, but their rate constant for formation of inner sphere complexes is more than 3 orders of magnitude higher. ${ }^{35}$ Thus, the parameters of polarization measured over a broad frequency range can be used as evidence for the state of ion binding.

\section{Ion binding in the protein}

The data obtained in the present investigation demonstrate that the dispersion of the polarizability is influenced by the presence of bivalent ions, but this effect is small compared with the strong influence of the ion composition on the limit dichroism $\xi_{\infty}$. In the absence of bivalent ions the $\xi_{\infty}$-value is much higher than in the presence of $\mathrm{Ca}^{2+}$ or $\mathrm{Mg}^{2+}$-ions. It is remarkable that the ion induced changes in the limit dichroism are much larger than those both in the polarizability and the permanent dipole moment. Thus, it is likely that the changes in the limit dichroism are not mainly due to ion binding at surface residues but probably involve ion binding sites close to the retinal group.

Binding sites for ions in the protein may be characterized by measurements with different mutants. The data obtained for the wild-type and D96N bacteriorhodopsin are very similar with respect to the frequency dependence of the polarizability, as should be expected for equivalent ionic atmospheres. However, there are some differences in the limit dichroism as a function of the frequency ( $c f$. Table 1), which should be characterized in more detail. Replacement of amino acid residues at different sites may be used for identification of binding sites.

\section{Bending/stretching of membrane fragments}

The state of polarization may also be affected by bending or stretching of the purple membrane fragments. Because the induced dipole of stretched states is expected to be larger than that of bent states, field induced stretching must be expected under conditions with a dominant induced dipole. The opposite process of bending may be induced by electric fields under conditions with a dominant permanent dipole, because bending of objects with a net charge increases their permanent dipole. Bending/stretching processes should be reflected by a special contribution to the dichroism. A modulation of the dichroism at $0.2 \mathrm{kHz}$ and high field strengths was observed, that may be partly due to bending/stretching. However, these data were not included in the evaluation of the polarizability. Thus, the polarizabilities in the range $\geq 0.2 \mathrm{kHz}$ should not be affected by bending/stretching, whereas the DC-polarizabilities are expected to contain a contribution from bending/ stretching processes. The large increase of the polarizability from $0.2 \mathrm{kHz}$ to DC may be partly due to these processes.

\section{Acknowledgements}

The technical assistance of Jürgen Wawrzinek is gratefully acknowledged.

\section{References}

1 E. R. Kandel, J. H. Schwartz and T. M. Jessell, Principles of Neural Science, McGraw-Hill, New York, 2000.

2 R. P. Plonsey and R. C. Barr, Bioelectricity-A quantitative Approach, Plenum, New York, 1991
3 J. P. Reilly, Applied Bioelectricity-From electrical stimulation to electropathology, Springer, 1998.

4 W. Stoeckenius and R. A. Bogomolni, Ann. Rev. Biochem., 1982, 52, 587-616.

5 Isr. J. Chem., 1995, 35, 193-515, special issue ed. M. Ottolenghi and M. Sheves.

6 U. Haupts, J. Tittor and D. Oesterhelt, Ann. Rev. Biophys. Biomol. Struct., 1999, 28, 367-399.

7 Biochim. Biophys. Acta, 2000, 1460, 1-239, special issue ed. J. K. Lanyi.

8 L. Keszthelyi, Biochim. Biophys. Acta, 1980, 598, 429-436.

9 Y. Kimura, A. Ikegami, K. Ohno, S. Saigo and Y. Takeuchi, Photochem. Photobiol., 1981, 33, 435-439.

10 Y. Kimura, M. Fujiwara and A. Ikegami, Biophys. J., 1984, 45, 615-625.

11 K. Barabas, A. Der, Z. Dancshazy, P. Ormos, L. Keszthelyi and M. Marden, Biophys. J., 1983, 43, 5-11.

12 L. D. Kahn and S. I. Tu, Biopolymers, 1984, 23, 707-718.

13 S. P. Stoylov, G. Todorov and A. Zhivkov, Bioelectrochem. Bioenerg., 1984, 12, 49-55.

14 E. Papp, G. Fricsovszky and G. Meszena, Biophys. J., 1986, 49, 1089-1100.

15 S. G. Taneva, N. Jordanova and I. B. Petkanchin, Biophys. Chem., 1992, 44, 91-97.

16 S. G. Taneva, G. Todorov, I. B. Petkanchin and S. P. Stoylov, Eur. Biophys. J., 1987, 14, 415-421.

17 H. I. A. Mostafa, G. Varo, R. Toth-Boconadi and L. Keszthelyi, Biophys. J., 1996, 70, 468-472.

18 D. Porschke, Biophys. J., 1996, 71, 3381-3391.

19 E. Fredericq and C. Houssier, Electric Dichroism and Electric Birefringence, Clarendon, Oxford, 1973.

20 D. Porschke, in Protein Ligand Interactions: Hydrodynamics and Calorimetry-A Practical Approach, ed. S. E. Harding and B. Z. Chowdhry, Oxford University Press, Oxford, 2001, pp. 197-221.

21 M. Mandel, Mol. Phys., 1961, 4, 489-496.

22 G. Schwarz, J. Phys. Chem., 1962, 66, 2636-2642.

23 J. M. Schurr, J. Phys. Chem., 1964, 68, 2407-2413.

24 J. P. McTague and J. H. Gibbs, J. Chem. Phys., 1966, 44, 4295-4301

25 F. Oosawa, Biopolymers, 1970, 9, 677-688.

26 G. S. Manning, Biophys. Chem., 1978, 9, 65-70.

27 D. C. Rau and E. Charney, Biophys. Chem., 1981, 14, 1-9.

28 M. Fixman, J. Chem. Phys., 1980, 72, 5177-5186.

29 M. Fixman and S. Jagannathan, J. Chem. Phys., 1981, 75, 4048-4059

30 W. van Dijk, F. van der Touw and M. Mandel, Macromol., 1981, 14, 792-795.

31 G. S. Manning, J. Chem. Phys., 1993, 99, 477-486.

32 U. Mohanty and Y. Zhao, Biopolymers, 1996, 38, 377-388.

33 J. Antosiewicz and D. Porschke, J. Phys. Chem., 1991, 95, 5983-5988.

34 J. Antosiewicz and D. Porschke, J. Phys. Chem. B, 1997, 101, 4478-4484.

35 H. Diebler, M. Eigen, G. Ilgenfritz, G. Maass and R. Winkler, Pure Appl. Chem., 1969, 20, 93-115.

36 S. P. Stoylov, Colloid Electro-Optics, Academic Press, 1991.

37 S. G. Taneva and I. B. Petkanchin, Trends Photochem. Photobiol., 1999, 6, 113-139.

38 S. G. Taneva, G. Todorov, I. B. Petkanchin and S. P. Stoylov, Eur. Biophys. J., 1987, 14, 415-421.

39 G. Wang and D. Porschke, J. Phys. Chem. B, 2003, 107, 4632-4638.

40 N. Grigorieff, T. A. Ceska, K. H. Downing, J. M. Baldwin and R. Henderson, J. Mol. Biol., 1996, 259, 393-421.

41 D. Oesterhelt and W. Stoeckenius, Methods Enzym., 1974, 31, 667-678.

42 M. J. Shah, J. Phys. Chem., 1963, 67, 2215-2219.

43 S. Diekmann, M. Jung and M. Teubner, J. Chem. Phys., 1984, 80, 1259-1262.

44 G. Schwarz, Z. Physik, 1956, 145, 563-584.

45 J. Antosiewicz, T. Grycuk and D. Porschke, J. Chem. Phys., 1991, 95, 1354-1360.

46 F. J. Perrin, Physique, 1934, 5, 497-511.

47 H. P. Schwan, G. Schwarz, J. Maczuk and H. Pauly, J. Phys. Chem., 1962, 66, 2626-2635.

48 D. Porschke, Biophys. Chem., 1985, 22, 237-247. 